\title{
47. On Bacterial Enzyme Specifically Decomposing Group B Substance
}

\author{
By Shoei Iseki and Tsukasa IkEDA \\ Department of Legal Medicine, School of Medicine, \\ Gunma University, Maebashi, Japan \\ (Comm. by T. Furuhata, M.J.A., March 12, 1956)
}

In 1951, Iseki and Okada ${ }^{1)}$ reported on an enzyme derived from Clostridium tertium and specifically decomposing group A substance. Later, Iseki and Tsunoda ${ }^{2)}$ reported on an enzyme derived from Bacillus fulminans and specifically decomposing $\mathrm{O}(\mathrm{H})$ substance. Further, Iseki and Masaki ${ }^{334)}$ clarified that these enzymes annihilated the group specificities by separating L-fucose from the group specific substances, and that the enzyme derived from Clostridium tertium, acting upon A substance, transformed it into $\mathrm{O}(\mathrm{H})$ substance. The present paper reports on a bacterium which has recently been isolated by the present authors from the soil, and which releases an enzyme specifically decomposing B substance.

\section{Materials and Methods}

1) Enzyme liquid. The bacterium was cultured in broth at $37^{\circ} \mathrm{C}$ for 48 hours, the culture liquid adjusted to $\mathrm{pH} 7.8$, centrifuged with a high speed centrifuge, and the supernatant was used as the enzyme liquid.

It was added to the same volume of the group substance solution, the whole adjusted to $\mathrm{pH} 7.8-8.2$, allowed to stand at $37^{\circ} \mathrm{C}$ for 48 hours, and heated in a water bath of $100^{\circ} \mathrm{C}$ for 5 minutes to destroy enzymic action. Then it was centrifuged, and the supernatant was subjected to an agglutinin absorption test and a group specific precipitation test to examine for the decomposition of the blood group substance.

The examination for the same object was also made by culturing bacterium in a synthetic minimal medium in which glucose was replaced by group $\mathrm{B}$ substance. The composition of the minimal medium was as follows: $\mathrm{K}_{2} \mathrm{HPO}_{4}, 7 \mathrm{~g}$; $\mathrm{KH}_{2} \mathrm{PO}_{4}, 3 \mathrm{~g}$; sodium citrate, $0.5 \mathrm{~g} ; \mathrm{MgSO}_{4} \cdot 7 \mathrm{H}_{2} \mathrm{O}, 0.1 \mathrm{~g} ;\left(\mathrm{NH}_{4}\right)_{2} \mathrm{SO}_{4}, 1 \mathrm{~g}$; glucose, $2 \mathrm{~g}$; distilled water, $1000 \mathrm{cc}$.

2) Blood group substances. Human secretor salivas of O, A, and B groups were used.

3) Antisera. As anti-O antibody, anti-O human blood cells immune chicken serum was used, as anti-A and anti-B precipitins, 
anti-A and anti-B human blood cells immune rabbit sera, respectively, and as anti-A and anti-B agglutinins, groups B and A human sera, respectively.

\section{Results}

1. Properties and identification of the bacterium. This bacterium is an aerobic bacillus, Gram-positive and motile, and has a central spore. The colony is circular, grayish white, and opaque, and has a rough surface. It grows by anaerobic culture on blood agar plate, producing hemolysis. Biochemically it was found to be catalasepositive, to reduce nitrates but not to form indole. It liquefies gelatine and coagulated serum, peptonizes milk and hydrolyzes starch. It ferments glucose, fructose, maltose, dextrin, and saccharose in a day, glycerol in 3 days but not lactose, galactose, arabinose, rhamnose, xylose, raffinose, mannitol, inulin, and inositol. These properties are identical with those of Bacillus cereus described in Bergey's Manual of Determinative Bacteriology. ${ }^{5)}$

2. Group specific substance decomposing action. When an enzyme derived from this bacterium was allowed to act upon each group specific substance, group B saliva lost anti-B agglutinin absorbing ability and precipitability against anti-B precipitin, but group $\mathrm{O}$ and $\mathrm{A}$ substances did not lose the absorbing ability and precipitability against anti-O and anti-A antibodies, respectively, as shown in Table I.

Table I. Enzymic action against group specific substances

\begin{tabular}{|c|c|c|c|c|c|c|c|c|c|c|c|}
\hline \multicolumn{2}{|c|}{ Reaction } & \multicolumn{5}{|c|}{ Agglutinin absorption } & \multicolumn{5}{|c|}{ Precipitation } \\
\hline \multicolumn{2}{|c|}{ Antigen dilution } & $1 / 80$ & $1 / 160$ & $/ 32$ & 640 & 1280 & $1 / 20$ & $1 / 40$ & $1 / 80$ & $1 / 160$ & $1 / 320$ \\
\hline \multicolumn{2}{|c|}{ Antiserum } & \multicolumn{5}{|c|}{ Anti-O chicken serum } & \multicolumn{5}{|c|}{ Anti-O chicken serum } \\
\hline \multirow{2}{*}{$\begin{array}{c}\text { Group } \mathrm{O} \\
\text { saliva }\end{array}$} & $\begin{array}{l}\text { Before } \\
\text { action }\end{array}$ & - & - & - & - & + & + & + & + & + & - \\
\hline & $\begin{array}{l}\text { After } \\
\text { action }\end{array}$ & - & - & - & - & + & + & + & + & + & - \\
\hline \multicolumn{2}{|c|}{ Antiserum } & \multicolumn{5}{|c|}{ Anti-A human serum } & \multicolumn{5}{|c|}{ Anti-A rabbit serum } \\
\hline \multirow{2}{*}{$\begin{array}{c}\text { Group A } \\
\text { saliva }\end{array}$} & $\begin{array}{l}\text { Before } \\
\text { action }\end{array}$ & - & - & - & - & + & + & + & + & - & - \\
\hline & $\begin{array}{l}\text { After } \\
\text { action }\end{array}$ & - & - & - & - & + & + & + & + & - & - \\
\hline \multicolumn{2}{|c|}{ Antiserum } & \multicolumn{5}{|c|}{ Anti-B human serum } & \multicolumn{5}{|c|}{ Anti-B rabbit serum } \\
\hline \multirow{2}{*}{$\begin{array}{c}\text { Group B } \\
\text { saliva }\end{array}$} & $\begin{array}{l}\text { Before } \\
\text { action }\end{array}$ & - & - & - & - & + & + & + & + & + & - \\
\hline & $\begin{array}{l}\text { After } \\
\text { action }\end{array}$ & + & $\dot{+}$ & + & + & + & - & - & - & - & - \\
\hline
\end{tabular}


These results indicate that an enzyme released by Bacillus cereus specifically decomposes group B substance.

3. Properties of the B-decomposing enzyme. To investigate the action power of this enzyme, it was allowed to act at various $\mathrm{pH}$ upon the group B substance in human secretor saliva. The result is represented in the figure.

The optimum $\mathrm{pH}$ was 7.8-8.2, and no reaction occurred below $\mathrm{pH}$ 5.0 and above $\mathrm{pH} 9.4$.

The optimum temperature at the optimum $\mathrm{pH}$ was $30-37^{\circ} \mathrm{C}$, but the reaction was effected sufficiently well at $20^{\circ} \mathrm{C}$. When the

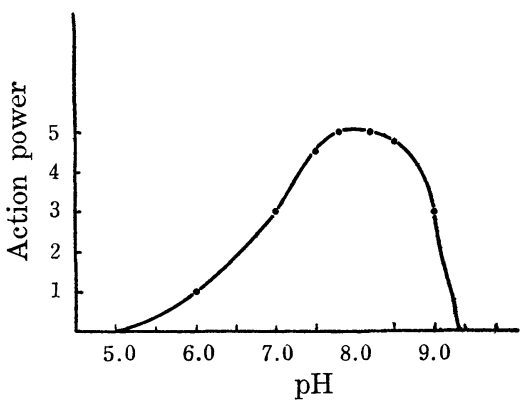

Relation between reaction power and reaction $\mathrm{pH}$ of enzyme enzyme liquid was heated at $50^{\circ} \mathrm{C}$ for 5 minutes, the enzymic action was considerably weakened. It was almost lost by heating at $60^{\circ} \mathrm{C}$ for 5 minutes, and completely lost at $70^{\circ} \mathrm{C}$ for 5 minutes.

4. Transformation from $\mathrm{B}$ to $\mathrm{O}(\mathrm{H})$ substance by enzyme. When Bacillus cereus was cultured in the above described synthetic medium containing $\mathrm{B}$ substance, the absorbing ability and precipitability against anti-B agglutinin and anti-B precipitin were lost, and those against anti-O agglutinin and anti-O precipitin were increased, as shown in Table II. However, group A specificity was not revealed in this case.

Table II. Action of B-decomposing enzyme against group B substance

\begin{tabular}{|c|c|c|c|c|c|c|c|c|c|c|c|}
\hline \multicolumn{2}{|c|}{ Reaction } & \multicolumn{5}{|c|}{ Agglutinin absorption } & \multicolumn{5}{|c|}{ Precipitation } \\
\hline \multicolumn{2}{|c|}{ Antigen dilution } & $1 / 80$ & \multicolumn{4}{|c|}{$1 / 160 \quad 1 / 320 \quad 1 / 640 \quad 1 / 1280$} & \multicolumn{5}{|c|}{$1 / 80 \quad 1 / 160 \quad 1 / 320 \quad 1 / 640 \quad 1 / 1280$} \\
\hline \multicolumn{2}{|c|}{ Antiserum } & \multicolumn{5}{|c|}{ Anti-O chicken serum } & \multicolumn{5}{|c|}{ Anti-O chicken serum } \\
\hline \multirow{2}{*}{$\begin{array}{c}\text { Group B } \\
\text { saliva }\end{array}$} & $\begin{array}{l}\text { Before } \\
\text { action }\end{array}$ & - & - & + & + & + & + & + & - & - & - \\
\hline & $\begin{array}{l}\text { After } \\
\text { action }\end{array}$ & - & - & - & - & + & + & + & + & + & - \\
\hline \multicolumn{2}{|c|}{ Antiserum } & \multicolumn{5}{|c|}{ Anti-B human serum } & \multicolumn{5}{|c|}{ Anti-B rabbit serum } \\
\hline \multirow{2}{*}{$\begin{array}{c}\text { Group B } \\
\text { saliva }\end{array}$} & $\begin{array}{l}\text { Before } \\
\text { action }\end{array}$ & - & - & - & - & + & + & + & - & - & - \\
\hline & $\begin{array}{l}\text { After } \\
\text { action }\end{array}$ & + & + & + & + & + & - & - & - & - & - \\
\hline
\end{tabular}


In the following experiment, Bacillus cereus was cultured in the synthetic medium containing group B saliva which was preliminarily acted upon by the O-decomposing enzyme from Bacillus fulminans. As shown in Table III, the agglutinin absorption test and group specific precipitation test revealed that the $O(H)$ specificity of $B$ saliva which was lost by the O-decomposing enzyme, was reproduced by the action of B-decomposing enzyme.

Table III. O- and B-decomposing enzymic actions against group B substance

\begin{tabular}{|c|c|c|c|c|c|c|c|c|c|c|}
\hline $\begin{array}{l}\frac{\text { Reaction }}{\text { Antigen dilution }} \\
\text { Antiserum }\end{array}$ & \multirow{3}{*}{\multicolumn{5}{|c|}{ 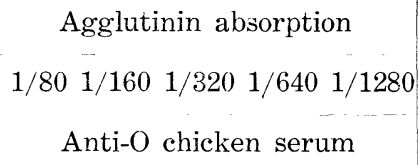 }} & \multicolumn{5}{|c|}{ Precipitation } \\
\hline \multirow{2}{*}{$\begin{array}{l}\text { Antigen dilution } \\
\text { Antiserum }\end{array}$} & & & & & & $1 / 40$ & $1 / 80$ & 160 & 320 & 640 \\
\hline & & & & & & & ti-O & ic & & \\
\hline Before action & - & - & + & + & + & + & + & + & - & - \\
\hline $\begin{array}{l}\text { Group B After action } \\
\text { saliva by O-enzyme }\end{array}$ & + & + & + & + & + & - & - & - & - & - \\
\hline $\begin{array}{l}\text { After action } \\
\text { by B-enzyme }\end{array}$ & - & - & - & + & + & + & + & + & + & - \\
\hline Antiserum & & ti-B & um & ser & & & ti- & & eru & \\
\hline Before action & - & - & - & - & + & + & + & + & - & - \\
\hline $\begin{array}{l}\text { Group B After action } \\
\text { saliva by O-enzyme }\end{array}$ & - & - & - & - & + & + & + & + & - & - \\
\hline $\begin{array}{l}\text { After action } \\
\text { by B-enzyme }\end{array}$ & + & + & + & + & + & - & - & - & - & - \\
\hline
\end{tabular}

The results indicate that the B-decomposing enzyme from Bacillus cereus transforms group B substance into $\mathrm{O}(\mathrm{H})$ substance by annihilating the group B specificity.

\section{Summary}

1. The authors discovered Bacillus cereus in the soil which produces an enzyme decomposing neither $\mathrm{O}(\mathrm{H})$ nor A substance but specifically B substance.

2. This enzyme can be almost destroyed by heating at $60^{\circ} \mathrm{C}$ for 5 minutes, and completely destroyed at $70^{\circ} \mathrm{C}$ for 5 minutes. The optimum $\mathrm{pH}$ for the enzymic action ranges $7.8-8.2$, and the optimum temperature at these $\mathrm{pH}, 30-37^{\circ} \mathrm{C}$.

3. The enzyme, acting upon $\mathrm{B}$ substance, transforms it into $\mathrm{O}(\mathrm{H})$ substance by annihilating its $\mathrm{B}$ specificity. 


\section{References}

1) Iseki, S., and Okada, S.,: On a specific enzyme which decomposes group A substances, Proc. Japan Acad., 27 (8), 455-458 (1951).

2) Iseki, S., and Tsunoda, S.,: On a bacterial enzyme which specifically decomposes O substance, Proc. Japan Acad., 28 (7), 370-373 (1952).

3) Iseki, S., and Masaki, S.,: Chemical actions of O- and A-specific enzymes on the respective blood group substances, Gunma J. Med. Sci., 4 (2), 105-116 (1955).

4) Iseki, S., and Masaki, S.,: Transformation of blood group substance by bacterial enzyme, Proc. Japan Acad., 29 (8), 460-465 (1953).

5) Breed, R. S., Murray, E. G. D., and Hitchens, A. P.,: Bergey's Manual of Determinative Bacteriology (Williams and Wilkins Company, Baltimore), 6th Ed., 715-717 (1948). 